\title{
28 Research Square \\ Cryo-EM structures reveal the electromotility mechanism of prestin, the cochlear amplifier
}

\section{Haon Futamata}

University of Tokyo

Masahiro Fukuda

University of Tokyo https://orcid.org/0000-0002-8824-846X

Rie Umeda

University of Tokyo

Keitaro Yamashita

University of Tokyo

Satoe Takahashi

Feinberg School of Medicine, Northwestern University

Takafumi Shikakura

Kyoto University

Shigehiko Hayashi

Kyoto University

\section{Kazuaki Homma}

Northwestern University https://orcid.org/0000-0002-4440-2277

\section{Tomohiro Nishizawa}

Yokohama City University

Osamu Nureki ( $\nabla$ nureki@bs.s.u-tokyo.ac.jp )

University of Tokyo https://orcid.org/0000-0003-1813-7008

\section{Article}

Keywords: cochlear amplification, electromotility, prestin, cryo-EM structures

Posted Date: October 1st, 2021

DOl: https://doi.org/10.21203/rs.3.rs-927386/v1

License: (1) This work is licensed under a Creative Commons Attribution 4.0 International License.

Read Full License 
Version of Record: A version of this preprint was published at Nature Communications on October 20th, 2022. See the published version at https://doi.org/10.1038/s41467-022-34017-x. 


\section{Abstract}

Outer hair cell electromotility, driven by prestin, is essential for mammalian cochlear amplification. Here, we report the cryo-EM structures of thermostabilized human prestin ( $\mathrm{hPres}^{\mathrm{TS}}$ ), complexed with chloride, sulfate, or salicylate at 3.52-3.61 A resolutions, revealing a crossed dimeric arrangement. The central positively-charged cavity allows flexible binding of various anion species, resulting in distinct modulations of nonlinear capacitance (NLC), playing an important role in electromotility. Comparisons of these $\mathrm{hPres}^{\mathrm{TS}}$ structures suggest rigid-body movement between the core and gate domains, and provide mechanistic insight into prestin inhibition by salicylate. Mutations at the dimeric interface severely diminished NLC, suggesting that stabilization of the gate domain facilitates core domain movement, thereby contributing to the expression of NLC. These findings advance our understanding of the molecular mechanism underlying mammalian cochlear amplification.

\section{Introduction}

Hearing is a process in which sound-induced mechanical vibrations are converted into electric signals within the cochlea and relayed to the brain. Two types of auditory hair cells, the inner (IHCs) and outer hair cells (OHCs), reside in the organ of Corti in the cochlea. The stereocilia of both hair cell types are mechanosensitive and transduce sound-induced mechanical displacement of the organ of Corti into changes in the receptor electric potential. IHCs are the direct sound sensory receptors that convert receptor potential changes into glutamatergic stimulation of the auditory nerves, whereas OHCs convert the receptor potential changes into somatic motility, which is referred to as $\mathrm{OHC}$ electromotility ${ }^{1}$. $\mathrm{OHC}$ electromotility mechanically amplifies the sound-induced displacement of the organ of Corti, which is otherwise attenuated by the viscous damping of the cochlear fluid. OHC electromotility plays an essential role in mammalian cochlear amplification, allowing humans to detect sound pressure as low as $20 \mu \mathrm{Pa}$.

Prestin (SLC26A5) is abundantly expressed in the lateral membrane of OHCs, where it is responsible for electromotility ${ }^{2,3}$. Prestin is unique in that it directly converts changes in the transmembrane electric potential into mechanical displacements. Unlike other SLC26 family members that function as anion transporters ${ }^{4}$, prestin's anion transport activity is very low ${ }^{5}$, implying that the voltage-driven motor function of prestin has evolved from an anion transport mechanism ${ }^{6}$. The motor activity of prestin coincides with the voltage-induced movement of voltage sensor charges, which manifests as nonlinear electric capacitance (NLC) ${ }^{7,8}$. Intracellular chloride ion is an essential co-factor of prestin ${ }^{9-11}$, but it remains unclear how chloride is held in prestin and contributes to the motor function. Salicylic acid inhibits the motor activity of prestin ${ }^{9}$, which accounts for the reversible hearing loss caused by aspirin overdosage ${ }^{12}$. Although recently solved structures of bacterial SLC26 ${ }^{13}$, mouse SLC26A9 ${ }^{14}$, and human SLC26A9 ${ }^{15}$ have allowed homology modeling of prestin with high confidence, many questions still remain regarding the voltage-driven motor mechanism of prestin. 
Here, we present the structures of thermostabilized prestin in complex with chloride, sulfate, and the prestin inhibitor, salicylate, determined by single-particle analyses using cryo-electron microscopy. These structures provide significant mechanistic insights toward the clarification of the voltage-driven motor function of prestin, which is responsible for the exquisite sensitivity and frequency selectivity of mammalian hearing.

\section{Results}

\section{Structure determination}

We performed single particle analyses of prestin proteins by cryo-EM to elucidate the voltagedependent motor mechanism of prestin. First, we expressed human prestin (hPres) with FLAG and GFP tags at the C-terminus in human embryonic kidney-293S (HEK-293S) cells, and after solubilization by digitonin, the protein was purified by affinity chromatography using FLAG antibody and GFP nanobodyimmobilized resins. The size-exclusion chromatography (SEC) analysis showed a broad peak corresponding to hPres, probably due to its partial aggregation during purification (Supplementary Fig. 1a). Consistently, the cryo-electron analysis revealed the highly heterogeneous particle shape and size of the purified hPres (Supplementary Fig. 1b), which provided only poor maps that were not sufficient to define the molecular architecture of hPres. To improve the structural stability, we engineered the thermostabilized hPres ( $\mathrm{hPres}^{\mathrm{TS}}$ ) by replacing multiple amino acid residues with those evolutionally conserved among prestin orthologs ${ }^{16,17}$ (Supplementary Fig. 2). This strategy allowed the purification of hPres $^{\text {TS }}$ in digitonin micelles with high homogeneity, as indicated by the excellent SEC elution profile (Supplementary Fig. 1c). The cryo-EM single particle analysis yielded a cryo-EM map of $\mathrm{hPres}^{\mathrm{TS}}$ at the overall resolution of $3.52 \AA$ (Fig. 1a-e), according to the gold-standard Fourier shell correlation (FSC 0.143) criterion (Supplementary Figs. 1d, 3, Supplementary Table 1).

The introduction of the TS mutations resulted in the loss of NLC within the experimentally measurable voltage range ( $\pm 200 \mathrm{mV}$ ) (Fig. 1f). Partial reversion of the TS mutations did not rescue NLC, and structural determinations of the 'partially reverted' $\mathrm{hPres}{ }^{\mathrm{TS}}$ constructs were unsuccessful. These observations suggest that the consensus mutations collectively stabilized the structure of $\mathrm{hPres}{ }^{\top \mathrm{T}}$.

\section{Overall Structure}

The structure of $\mathrm{hPres}^{\mathrm{TS}}$ protein forms a domain-swapped homodimer, with each protomer consisting of 14 transmembrane (TM) a-helices and the C-terminal cytosolic STAS domain crossing each other. Both the TM and C-terminal domains contribute to the dimer formation (Fig. 1a, b). The TM domain is divided into two subdomains: the core (TM helices 1-4 and 9-11) and the gate (TM helices 5-8 and 1214) domains (Fig. 1c, d). The inter-domain interface contains mostly hydrophobic residues. These structural observations indicate that the TM domain of the prestin protomer adopts the typical inverted repeat transporter fold ${ }^{14,15,18,19}$ which presently adopts an inward-open conformation. The overall structure remarkably resembles the cryo-EM structures of the mouse and human SLC26A9 anion 
transporters (Supplementary Fig. 6) ${ }^{14,15}$, although these two SLC26A family members have distinct physiological functions. SLC26A9 mediates channel-like fast anion transport ${ }^{20,21}$, whereas prestin (SLC26A5) has voltage-driven motor activity but barely detectable transport activity.

\section{Anion binding site}

The substrate transport by the SLC26A transporters is considered to follow an alternate access mechanism, in which the substrate binding site is alternately opened toward either side of the membrane, and passively driven by the elevator-like motions of the core and gate domains ${ }^{14,15}$. By analogy to other similarly folded transporters such as $\mathrm{UraA}^{19}$ and band $3^{18}$, the substrate binding site is presumably located at the pseudo-symmetric center of the core domain. The amino-terminal ends of the symmetrically arranged unwound helices (TM3 and TM10) are oriented toward this site, creating an electrically favorable environment for capturing negatively charged substrates between the dipoles (Fig. 1e, 2a). In fact, we observed a density at this site, which probably corresponds to a chloride ion coordinated by Arg399 and Ser396 (Fig. 2a). It is likely that Phe137 contributes to confining the chloride ion at the site (Fig. 2a). Arg399 is a highly conserved residue within the SLC26A family, and thus is expected to participate in the anion binding ${ }^{22,23}$. To investigate the functional roles of these residues located at the chloride binding site, we mutated them and measured the NLC (Fig. 2C). S396A abolished the NLC, while F137A largely shifted the voltage operating point of NLC to the depolarizing direction. These results affirmed the contributions of these residues for creating a favorable environment for anion binding and their importance for the generation of NLC. On the other hand, S396D showed wild-type-like NLC, probably because the negative charge of Asp mimics chloride binding. We also examined the roles of Ser116 and Ser133, which likely interact with Arg399 electrostatically, by mutating these residues to Glu or Asp (i.e., S116D, S116E, S133D, and S133E). As shown in Fig. 2c, all these mutations abolished NLC, indicating that, while Arg399 probably has the most important role in anion binding, several surrounding residues contribute to create an optimal environment. The nullified NLC of these mutants is not due to impaired membrane targeting, as a membrane-impermeable fluorescent dye successfully labeled (Supplementary Fig. 7). The stable anion binding at this positively charged cavity was confirmed by MD simulations for $100 \mathrm{nsec}$, using these inward-open structures: the anion was held in the cavity in balance with Arg399, the N-termini of symmetrical TMs and other surrounding residues (Supplementary Fig. 8a-c).

Since the replacement of chloride with sulfate largely shifts the voltage operating point $\left(\mathrm{V}_{\mathrm{pk}}\right)$ of prestin towards the depolarizing direction ${ }^{11,24}$, we next performed a cryo-EM analysis of hPres ${ }^{T S}$ in the presence of sodium sulfate. The overall structure of sulfate-bound $\mathrm{hPres}^{\mathrm{TS}}$ was essentially the same as the chloride-bound form of hPres $^{T S}$. However, as compared to the chloride ion that occupies the pseudosymmetrical center of the prestin molecule, coordinated by the Arg399 side chain and the N-termini of the TM3/TM10 helix dipoles (Fig. 2a), the bound sulfate ion was not present in this center and more exposed to the intracellular side (Fig. 2b), probably due to the differences in the anion size and charge valence. It is 
conceivable that the known dependence of NLC on various anion species $9,11,24$ can be partly ascribed to subtle differences in the binding positions among anions.

\section{Inhibition by salicylate}

Salicylate is a well-known inhibitor of $\mathrm{OHC}$ electromotility $25-27$, and directly acts on prestin ${ }^{9}$. It is likely that the salicylate-bound state mimics an elongated state ${ }^{28}$, because the application of salicylate induces the elongation of $\mathrm{OHCs}$ along the longitudinal direction (Supplementary Movie 1, Supplementary Fig. 9a). To understand the mechanism of prestin inhibition by salicylate, we performed a cryo-EM analysis of $\mathrm{hPres}^{\mathrm{TS}}$ in the presence of sodium salicylate. We observed a flat-shaped density at the interface between the core and gate domains, which likely corresponds to salicylate (Fig. 3a). The binding position of the polar moiety of salicylate is similar to that of sulfate, while the benzene ring moiety interacts hydrophobically with Phe137 and Val353, which likely inhibits the relative motion of the core/gate domains and freezes the structure in a certain state. The carboxylate moiety of salicylate was found in the proximity of Ser396 and Ser398, implying the contributions of these small polar residues for salicylate binding. Conversions of these residues to acidic ones (e.g., S396D/E and S398D/E) reportedly made prestin constitutively active and insensitive to inhibition by salicylate ${ }^{29,30}$. We confirmed that prestin $^{\mathrm{S396D}}$ and prestin ${ }^{\mathrm{S} 396 \mathrm{E}}$ are indeed insensitive to salicylate (Supplementary Fig. 9b). These "Argneutralization mutants" are likely to mimic the anion binding and thus prevent salicylate binding. We found that S398A also makes prestin insensitive to salicylate, further affirming the importance of the small polar residue for salicylate binding (Fig. 3b, Supplementary Fig. 9b).

Although the chloride- and salicylate-bound structures both adopt inward-open conformations, comparisons of these two cryo-EM structures revealed a small relative rigid body displacement between the core and gate domains (Fig. 3c, Supplementary Movie 2), which is consistent with the presumed elevator-like motion of transporters ${ }^{14,15,31}$. To investigate the role of this motion, we focused on GIn97, Asn447 and Glu293, as they are likely to contribute to the formation of hydrogen bonding interactions between the gate and core domains (Fig. 3d-f). Gln97 and Asn447 partly constitute the chloride binding site, and their mutations, Q97D and N447D, almost completely abolished NLC (Fig. 3g). In addition, the charge-reversing mutation of Glu293 (E293K), which is farther from the chloride binding site, also abolished NLC. We examined the role of a hydrophobic residue, Val353, which is near the anion binding site and exposed to the core/gate domain interface. We found that the conversion of this hydrophobic residue to hydrophilic ones (V353D, V353E, and V353N) drastically reduced or abolished NLC, whereas substitutions to other hydrophobic residues with different sizes (V353A and V353I) did not (Fig. 3g). These results suggest that the electrostatic and hydrophobic interactions, mediated by these residues located at the core/gate domain interface, facilitate an elevator-like motion and are important for NLC generation.

\section{Dimerization of prestin}


A pivotal hydrophobic interface formed by Val499 and lle500 (mutated to Leu in hPres $^{\mathrm{TS}}$ ) is located at the C-terminus of the long TM14 helix and involved in the domain-swapped dimerization interface of prestin (Fig. 4a). Mutations that attenuate the hydrophobicity of these residues, such as V499G and I500G, almost completely abolished NLC (Fig. 4b), in agreement with previous reports ${ }^{32,33}$. Even a modest mutant $(\mathrm{I500 \textrm {L }})$ notably affected the voltage sensitivity $\left(0.032 \pm 0.006 \mathrm{mV}^{-1} \mathrm{vs} .0 .017 \pm\right.$ $\left.0.001 \mathrm{mV}^{-1}, p<0.0001\right)$ and voltage operating point $(-65 \pm 14 \mathrm{mV}$ vs. $-22 \pm 8.5 \mathrm{mV}, p<0.0001)$ of prestin (WT, $n=22$ vs. 1500L, $n=5$, mean $\pm S D$ ) (Fig. 4b), indicating that stable domain-swapped dimer formation is crucial for NLC generation. It is likely that an elevator-like motion of the core domain underlies NLC, and the rigid dimerization of the gate domains facilitates this conformational transition. We also observed strong densities of lipid acyl chains and cholesterols on the gate domains (Fig. 4c, d), further suggesting the importance of their stabilization. This observation also provides insight into the known effects of lipid manipulation on the function of prestin ${ }^{34-36}$.

\section{Discussion}

In this study, we observed hPres ${ }^{\top S}$ structures in inward-open conformations. Although SLC26A transporters are thought to alternate between inward- and outward-open conformations, mammalian prestin orthologs may not assume an outward-open conformation ${ }^{37}$. It is probably because electric attraction between the inner positively-charged central cavity of prestin and membrane potential. However, it is conceivable that the rigid body motions of the core and gate domains, which would be similar to the elevator-like motions presumed for other SLC26A anion transporters ${ }^{14,15,22}$, occur in a voltage-dependent manner in prestin (Fig. 5), and that such conformational changes are intimately linked to the electromotility and concurrent NLC. Arg399 and the symmetrically arranged N-termini of the TM3 and TM10 helices provide a strong, positively charged electric field that attracts anions at the center of the core domain (Fig. 2). It is unlikely that such a highly charged cavity is isolated from the bulk solvent without being neutralized by a counter charge (Fig. 5a). Therefore, anion binding to the positively charged cavity within the prestin protein would be essential for the transition from the inward-open state to the putative occluded state, in which the anion binding site is isolated from both extracellular and intracellular solvents (Fig. 5a, b). Our MD simulation supported the stable accommodation of a chloride ion in the positively charged cavity (Supplementary Fig. 8a-c). Mutations that result in the neutralization of this positively charged local environment, such as S396D and S396E, likely mimic chloride-bound states, thereby allowing the conformational transitions of prestin. Notably, the coordination distance between the chloride ion and Arg399 found in our cryo-EM hPres ${ }^{\top S}$ structure is $4.49 \AA$, which is longer than the typical coordination distances of chloride ions found in chloride-bound protein structures deposited in the PDB server ${ }^{38}$. This unusually long coordinate distance can be ascribed to the additional positive electrical fields created by the helix dipoles of TM3 and TM10. Salicylate binds to this positively charged area with its carboxylate mimicking the anionic moiety, but the aromatic benzene ring protrudes toward the gate domain and thereby sterically restricts the elevator-like motion of the core/gate domains (Figs. 3f, 5c). These observations suggest that the bound anion and surrounding environment are likely to 
serve as the extrinsic voltage sensor in prestin, and their longitudinal migration due to the elevator-like movement might be detected as NLC 9,39,40, while other charged residues in the core domain also contribute to the net charge movement.

Our mutational analyses focused on the residues located at the gate/core domain interface (Fig. 3D-G), suggesting the presence of a hydrogen-bonding network that affects the putative voltage-driven, relative rigid body motions between the two domains by bridging them. It is structurally possible for anion binding to affect this hydrogen bonding network to facilitate the rigid body motion. We found that the chloride and sulfate binding sites are distinct (Figs. 2 and 3), probably due to the differences in their sizes and charge valences. It is thus likely that the hydrogen bonding network, which probably affects the elevator-like movement of the core/gate domains, is distinctly affected by different anions, thereby accounting for the known anion dependence of NLC. Collectively, we propose the dual functional significance of anion binding to prestin for its voltage-driven motor function. Firstly, considering the positive inside rule, neutralization of the positively charged central cavity by anion binding is essential for allowing prestin to transit to the putative occluded state (Fig. 5B). Secondly, modulation of the hydrogen bonding network by a bound anion is requisite for the rigid body motions of the core domain with respect to the gate domain. Furthermore, depolarization may facilitate the rigid body motion due to the partlyneutralized positively charged central cavity. These effects cooperatively allow the voltage-dependent conformational change of the core domain of prestin. Since prestin is highly unlikely to function without an extrinsic anion, the possibility that the bound anion also contributes to voltage sensing ${ }^{9,39}$ should not be disregarded.

The voltage-dependent conformational changes of prestin molecules are likely to be directly converted into $\mathrm{OHC}$ contraction and elongation, which can be up to $\sim 5 \%$ of the total length of an $\mathrm{OHC}^{41,42}$. We speculate that the putative voltage-induced elevator-like motions of the core domains underlie this electromechanical conversion. We also surmise that rigid dimer formation is important for the voltagedriven motor function of prestin. The cryo-EM structure of hPres ${ }^{\top \mathrm{TS}}$ suggested highly stable dimer formation, mediated by hydrophobic residues at the C-terminal ends of TM14, hydrophilic residues located at the interface of the STAS domains, and multiple lipids filling the dimeric junction region (Figs. 1 and 4). In fact, the V499G and I500G mutations, which are expected to destabilize the dimeric interaction between prestin protomers, abolished NLC (Fig. 4b). Rigid dimerization-assisted fixation of the orientation of the gate domains, with respect to the axis perpendicular to the membrane plane, might be crucial for making the yet to be identified voltage sensor of prestin optimally responsive to changes in the transmembrane electric potential, and thus for efficiently converting the motions of the core domains into molecular motility. The elevator-like motions of the core domains with respect to the homodimeric gate domains with two-fold symmetry probably produce the directional motor activity. Therefore, understanding how prestin molecules are connected to the submembrane structure is crucial, in order to fully appreciate the molecular and cellular mechanisms of $\mathrm{OHC}$ electromotility responsible for the exquisite sensitivity and frequency selectivity of mammalian hearing. 


\section{Methods}

\section{Plasmids preparation}

Full-length human prestin (hPres) was amplified using human universal reference cDNA (Zyagen) as the template, and cloned into a modified pEG BacMam vector with a C-terminal TEV cleavage site followed by a FLAG-EGFP tag. A thermostabilized prestin protein (hPres ${ }^{\mathrm{TS}}$ ) was designed by the consensus mutagenesis approach, based on the sequences of all eukaryotic prestin orthologs (https://github.com/TaizoAyase/consensus_creator)_ ${ }^{17}$. About $40 \%$ of the amino acid residues were altered in hPres $^{T S}$ as compared to wild-type hPres (Supplementary Fig. 2). A cDNA encoding hPres ${ }^{T S}$ was synthesized with codons optimized for expression in human cell lines, and cloned into the modified pEG BacMam vector described above. For functional assays, naked mole rat (Heterocephalus glaber) prestin (HgPres) was used for better expression as compared to hPres. The amino-acid sequence of HgPres is $96 \%$ identical to that of hPres (Supplementary Fig. 2). Wild-type HgPres cDNA was synthesized based on the mRNA sequence (XM_004839711.2) by General Biosystems (Durham, NC), and cloned into the pSBtet-Pur vector ${ }^{43}$ with a C-terminal mTurquoise2 (mTq2) tag. Standard mutagenesis methods were used to generate HgPres mutants in the pSBtet-Pur vector.

\section{Purification of hPres and hPres $\underline{\text { TS }}$}

Baculoviruses carrying the prestin constructs were produced and amplified in Sf9 cells, using the Bac-toBac system (Invitrogen). HEK293S $\mathrm{GnTl}^{-}$cells (ATCC, CRL-3022) were grown and maintained in FreeStyle 293 medium (Gibco) at $37^{\circ} \mathrm{C}$ with $8 \% \mathrm{CO}_{2}$. For protein expression, the baculovirus was added at 1/10 $(\mathrm{v} / \mathrm{v})$ to the culture medium of HEK293S $\mathrm{GnTI}^{-}$cells, at a density of $3 \times 10^{6} \mathrm{cells} / \mathrm{mL}$. After 16-18 hrs, 5 $\mathrm{mM}$ valproic acid was added, and the cells were further incubated at $30^{\circ} \mathrm{C}$ with $8 \% \mathrm{CO}_{2}$ for $48 \mathrm{hrs}$. The cells were collected by centrifugation $\left(5,000 \times \mathrm{g}, 12 \mathrm{~min}, 4^{\circ} \mathrm{C}\right)$ and lysed by sonication in buffer, containing $50 \mathrm{mM}$ Tris- $\mathrm{HCl}, \mathrm{pH} 8.0,300 \mathrm{mM} \mathrm{NaCl}$, and protease inhibitors $(1.7 \mu \mathrm{g} / \mathrm{mL}$ aprotinin, 0.6 $\mu \mathrm{g} / \mathrm{mL}$ leupeptin, $0.5 \mu \mathrm{g} / \mathrm{mL}$ pepstatin and $1 \mathrm{mM}$ PMSF). After cell debris removal by centrifugation $\left(4,000 \times \mathrm{g}, 12 \mathrm{~min}, 4^{\circ} \mathrm{C}\right)$, the membrane fraction was collected by ultracentrifugation $(186,000 \times \mathrm{g}, 1 \mathrm{hr}$, $4^{\circ} \mathrm{C}$ ). The membrane fraction was solubilized in buffer, containing $50 \mathrm{mM}$ Tris- $\mathrm{HCl}, \mathrm{pH} 8.0,300 \mathrm{mM} \mathrm{NaCl}$, 10\% glycerol, 2 mM 2-mercaptoethanol ( $\beta$-ME), and 1\% digitonin (Calbiochem), for $1 \mathrm{hr}$ at $4^{\circ} \mathrm{C}$. After ultracentrifugation $\left(186,000 \times \mathrm{g}, 20 \mathrm{~min}, 4^{\circ} \mathrm{C}\right)$, the supernatant was collected and incubated with antiFLAG M2 affinity gel (Sigma) for $1 \mathrm{hr}$ at $4^{\circ} \mathrm{C}$. The resin was washed with 10 column volumes of wash buffer, containing $50 \mathrm{mM}$ Tris- $\mathrm{HCl}, \mathrm{pH} 8.0,300 \mathrm{mM} \mathrm{NaCl}, 20 \%$ glycerol, $2 \mathrm{mM} \beta-\mathrm{ME}$, and 0.1\% digitonin. Bound prestin was eluted with the wash buffer containing $0.125 \mathrm{mg} / \mathrm{mL}$ of FLAG peptide, and EGFP fluorescence positive elution fractions were collected. For further purification, CNBr-Activated Sepharose 4 Fast Flow Beads (GE Healthcare) conjugated with anti-GFP nanobodies ${ }^{44}$ were added to the collected fractions and incubated for $1 \mathrm{hr}$ at $4^{\circ} \mathrm{C}$. The resin was washed with 10 column volumes of the same wash buffer and then gently suspended with TEV protease (purified in-house) overnight at $4^{\circ} \mathrm{C}$ to cleave the Cterminal FLAG-EGFP tag. After the TEV protease cleavage, the flow-through fraction was pooled, 
concentrated to $5-10 \mathrm{mg} / \mathrm{mL}$ using a centrifugal filter device (Millipore $100 \mathrm{kDa}$ MW cutoff) and loaded onto a Superose 6 Increase 10/300 GL column (GE Healthcare), equilibrated in buffer containing $50 \mathrm{mM}$ Tris- $\mathrm{HCl}, \mathrm{pH} 8.0,300 \mathrm{mM} \mathrm{NaCl}, 2 \mathrm{mM} \beta-\mathrm{ME}$, and $0.1 \%$ digitonin. The peak fractions were pooled and concentrated to $5-10 \mathrm{mg} / \mathrm{mL}$.

\section{Electron microscopy sample preparation}

After precipitating aggregated proteins by ultracentrifugation at $138,000 \times \mathrm{g}$ for $20 \mathrm{~min}$, a $3 \mu \mathrm{L}$ portion of the supernatant was spotted onto a glow-discharged holey carbon grid (Quantifoil R1.2/1.3, Cu/Rh, 300 mesh), which was plunge-frozen in liquid ethane using a Vitrobot Mark IV (FEI) at $6^{\circ} \mathrm{C}$ with a blotting time of $4 \mathrm{sec}$ with $100 \%$ humidity. To obtain sulfate- and salicylate-bound $\mathrm{hPres}^{\mathrm{TS}}$, the purified protein solution was mixed with sodium sulfate $(10 \mathrm{mM})$ or sodium salicylate $(30 \mathrm{mM})$, respectively, and incubated on ice for $15 \mathrm{~min}$ before grid preparation.

\section{Electron microscopy data collection and processing}

The prepared grids were transferred to a Titan Krios G4 microscope (Thermo Fischer Scientific), equipped with a Gatan Quantum-LS Energy Filter (GIF) and a Gatan K3 Summit direct electron detector. The camera was operated in the correlated double sampling (CDS) mode. The dataset was collected at a nominal magnification of $\times 105,000$, corresponding to a calibrated pixel size of $0.83 \AA$ per pixel (The University of Tokyo, Japan). Each movie was recorded for 5.0 seconds and subdivided into 64 frames. The electron flux rate was set to $7.5 \mathrm{e}^{-} / \mathrm{pix} / \mathrm{s}$ at the detector, resulting in an accumulated exposure of $54 \mathrm{e}^{-} / \AA^{2}$ at the specimen. The data were automatically acquired using the SerialEM software ${ }^{45}$, with a defocus range of -0.8 to $-1.6 \mu \mathrm{m}$.

Initially, all datasets were corrected for beam-induced motion, using the motion correction program implemented in the single-particle analysis software RELION-3.1 ${ }^{46}$, and the contrast transfer function (CTF) parameters were estimated using CTFFIND4 ${ }^{47}$. For the dataset of the chloride-bound state, 1,373,022 particles were picked from 4,680 micrographs by using the Laplacian-of-Gaussian picking function in RELION-3.1, and were used to generate two-dimensional (2D) models for reference-based particle picking. Particles were extracted with down-sampling to a pixel size of $3.63 \AA / p i x$ and subjected to several rounds of 2D and 3D classifications. The best class contained 341,744 particles, which were then re-extracted with a pixel size of $1.10 \AA /$ pix and subjected to 3D refinement. The resulting 3D model and particle set were subjected to per-particle CTF refinement, beam-tilt refinement, Bayesian polishing ${ }^{48}$, and 3D refinement. The final 3D refinement and postprocessing of the three classes yielded maps with global resolutions of $3.52 \AA$, according to the $\mathrm{FSC}=0.143$ criterion ${ }^{49}$. The local resolution was estimated using RELION-3.1. The processing strategy is described in Supplementary Fig. 3. For the dataset of the sulfate-bound state, 1,181,278 particles were picked from 4,077 micrographs, and the best class containing 249,144 particles was selected and processed as above, yielding a map with a global resolution of $3.52 \AA$ (Supplementary Fig. 4). For the dataset of the salicylate-bound state, 848,704 particles were picked from the 3,375 micrographs by reference-based particle picking with the map of the 
chloride-bound state. The best class containing 113,410 particles was selected and processed, yielding a map with a global resolution of $3.57 \AA$ (Supplementary Fig. 5).

\section{Model building and validation}

The models of the chloride-bound state of $\mathrm{hPres}^{\mathrm{TS}}$ were manually built de novo in the Cryo-EM density map in COOT ${ }^{50}$, facilitated by the previously reported crystal structures of SLC26Dg (PDB ID: 5DA0). Structure refinement was initially performed with Rosetta ${ }^{51}$ and phenix.real_space_refine ${ }^{52,53}$. After manual adjustments, the models were then subjected to structure refinement with the Servalcat pipeline using REFMAC5 ${ }^{54,55}$ and manual real-space refinement in COOT ${ }^{50}$. The models of the salicylate-bound state were built by using the chloride-bound model as the starting model. The statistics of the 3D reconstruction and model refinement are summarized in Supplementary Data Table 1. All molecular graphics figures were prepared with CueMol (http://www.cuemol.org).

\section{$\underline{\text { NLC measurement }}$}

HEK293T-based stable cell lines expressing HgPres-mTq2 constructs in a doxycycline-dependent manner were established and maintained in DMEM, supplemented with $10 \% \mathrm{FBS}$ and $1 \mu \mathrm{g} / \mathrm{ml}$ puromycin (Fisher Scientific), as previously described ${ }^{56,57}$. The expression of the HgPres constructs was induced by adding $1 \mu \mathrm{g} / \mathrm{mL}$ doxycycline to the culture medium, one day prior to NLC recording. Whole-cell NLC recordings were performed at room temperature, using an Axopatch 200B amplifier (Molecular Devices, Sunnyvale, CA). Recording pipettes were pulled from borosilicate glass to achieve initial bath resistances averaging 3-4 $\mathrm{M} \Omega$. Recordings were performed using sinusoidal (2.5- $\mathrm{Hz}, 120-150 \mathrm{mV}$ amplitude) voltage stimuli superimposed with two sinusoidal stimuli (390.6 (f1) and 781.3 (f2) $\mathrm{Hz}, 10 \mathrm{mV}$ amplitude). Recording pipettes were filled with an intracellular solution containing $140 \mathrm{mM} \mathrm{CsCl}, 2 \mathrm{mM} \mathrm{MgCl}_{2}, 10 \mathrm{mM}$ EGTA, and $10 \mathrm{mM}$ HEPES ( $\mathrm{pH}$ 7.3). Cells were bathed in an extracellular solution containing $120 \mathrm{mM} \mathrm{NaCl}, 20$ $\mathrm{mM}$ TEA-Cl, $2 \mathrm{mM} \mathrm{CoCl} 2,2 \mathrm{mM} \mathrm{MgCl}_{2}$, and $10 \mathrm{mM} \mathrm{HEPES} \mathrm{(pH} \mathrm{7.3).} \mathrm{Osmolality} \mathrm{was} \mathrm{adjusted} \mathrm{to} 304$ $\mathrm{mOsmol} / \mathrm{kg}$ with glucose. Current data were collected and analyzed by jClamp (SciSoft Company, New Haven, CT) ${ }^{58}$.

NLC $\left(C_{m}\right)$ data were analyzed using the following equation:

$$
C_{m}=\frac{\alpha Q_{\max } \exp \left[\alpha\left(V_{m}-V_{p k}\right)\right]}{\left\{1+\exp \left[\alpha\left(V_{m}-V_{p k}\right)\right]\right\}^{2}}+C_{l i n}
$$

where $a$ is the slope factor, $Q_{\max }$ is the maximum charge transfer, $V_{m}$ is the membrane potential, $V_{p k}$ is the voltage at which the maximum charge movement is attained, and $\mathrm{C}_{\text {lin }}$ is the linear capacitance. The magnitude of NLC $\left(C_{m}-C_{\text {lin }}\right)$ was corrected for cell size $\left(C_{\text {lin }}\right)$ because larger cells tend to express greater amounts of the prestin protein $\left(\mathrm{C}_{\mathrm{sp}} \equiv\left(\mathrm{C}_{\mathrm{m}}-\mathrm{C}_{\text {lin }}\right) / \mathrm{C}_{\text {lin }}\right)$. 
Stable cells were seeded on a 6-well plate, and the expression of mTq2-tagged HgPres constructs was induced by $3 \mu \mathrm{g} / \mathrm{mL}$ doxycycline for 2 days prior to labeling. Cells were washed once with PBS, and $2 \mathrm{~mL}$ of $10 \mu \mathrm{M}$ Sulfo-Cyanine3 NHS ester (Lumiprobe) dissolved in ice-cold PBS was added (per well) and incubated for 30 minutes at $4{ }^{\circ} \mathrm{C}$. The reaction was stopped by the addition of $200 \mu \mathrm{L}$ of $100 \mathrm{mM}$ glycine. Cells were collected and lysed by sonication on ice in $500 \mu \mathrm{L}$ of lysis buffer $(150 \mathrm{mM} \mathrm{NaCl}, 20 \mathrm{mM}$ HEPES, pH 7.5, 1 mM EDTA, 20 mM DDM, 1 mM DTT, and $50 \mu \mathrm{g} / \mathrm{mL}$ leupeptin). The lysate was centrifuged at $16,000 \times \mathrm{g}$ for 5 minutes at $4^{\circ} \mathrm{C}$. A GFP selector slurry ( $5 \mu \mathrm{L}$, NanoTag Biotechnologies) was added to the supernatant and incubated for 30 minutes at $4{ }^{\circ} \mathrm{C}$, with end-over-end mixing using a rotator. Bound proteins were collected alongside with the GFP selector by brief centrifugation, and observed with a fluorescent microscope (Leica DMIRB) controlled by $\mu$ Manager ${ }^{59}$. Merged images of GFP selectors in cyan and red channels were analyzed using $\mathrm{FIJI}^{60}$ to determine the fluorescent signal intensities of mTq2 and Cy3. The results are shown in Supplementary Fig. 7.

\section{Molecular dynamics simulation}

We performed molecular dynamics (MD) simulations to analyze interactions of a chloride ion ( $\left.\mathrm{Cl}^{-}\right)$ binding to prestin. Wild-type hPres model was constructed by modeller ${ }^{61}$, using the chloride-bound hPres ${ }^{\text {TS }}$ cryo-EM structure as the template. The system includes residues from Arg58 to Pro506. Hydrogen atoms were added using the psfgen plugin in NAMD. The protonation states of ionizable residues were assumed to be those at $\mathrm{pH} 7$ by PROPKA. The structure of prestin was manually embedded in a $105 \AA$ × $105 \AA$ 1-palmitoy-2-oleoyl-sn-glyceroo-3-phosphocholine (POPC) bilayer modeled with VMD. The bilayer was solvated with TIP3P water molecules with $\mathrm{Na}^{+}$and $\mathrm{Cl}^{-}$ions of $0.15 \mathrm{M}$ using the solvate plugin and the autoionize plugin in VMD. The simulation box was initially $105 \AA$ × $105 \AA$ × $120 \AA$ in size and contained 110,295 atoms.

We first performed a preparatory MD simulation with the NAMD program package. The force field parameters of the protein, water molecules, and lipids were CHARMM27, TIP3P, and CHARMM36, respectively. Short-range non-bonded interactions were cutoff at $12 \AA$ with a force-switching function. Long-range electrostatic interactions under periodic boundary conditions were calculated with the particle mesh Ewald method. Temperature and pressure were controlled with Langevin dynamics and the Langevin piston method, respectively. In regulation of the pressure, the $x$ and $y$ axes were isotropically scaled (NPTiso), where the $z$ axis was perpendicular to the membrane surface. The initial degrees of freedom of water molecules were constrained with the SETTLE algorithm. Bond including hydrogen atom were constrained with the RATTLE method. First, a 1,000-step energy minimization under the NVT condition were performed. Then, a 160-ps heating to $200 \mathrm{~K}$ under the NVT condition and a 40-ps heating simulation to $300 \mathrm{~K}$ under the NPTiso condition with the protein, water molecules, and lipid tails only being allowed to move. The time step of trajectory calculation was set to $2 \mathrm{fs}$. After 10-ns equilibrium MD simulation under NPTiso condition with the water molecules, and lipids tails only being allowed to move, 10-ns equilibrium MD simulation under NPTiso condition without any restraint was performed. The force field parameters were then switched to those of the Amber force field (ff14SB, lipid17, and TIP3P for the 
protein, lipids, and water molecules, respectively), and a 50-ns equilibrium simulation was carried out with NAMD. Finally, from the last snapshot of the equilibrated MD system obtained by the preparatory simulation described above, a sampling MD simulation for $100 \mathrm{~ns}$ was performed with pmemd.cuda.MPI of the AMBER16 program package with the Amber force fields (ff14SB, lipid14, and TIP3P for the protein, lipids, and water molecules, respectively).

A stable binding of the $\mathrm{Cl}^{-}$ion in the binding pocket was observed during the MD simulation for $100 \mathrm{~ns}$ (Supplementary Fig. 8). It is noteworthy that the binding of the $\mathrm{Cl}^{-}$ion in the binding pocket was not achieved by tight binding with the protein side-chains in the binding pocket such as Arg399, Ser396, and Ser398. The $\mathrm{Cl}^{-}$ion was not directly attached to Arg399 (Supplementary Fig. 8b), and the hydroxy groups of Ser396 and Ser398 frequently detached from the $\mathrm{Cl}^{-}$ion (Supplementary Fig. 8c). The stable accommodation of the $\mathrm{Cl}^{-}$ion in the binding pocket without the strict recognition of the $\mathrm{Cl}^{-}$ion by tight binding of the protein groups implies that the $\mathrm{Cl}^{-}$ion binding is mainly fulfilled by a strongly positive electrostatic environment at the binding pocket created by a positive charge of Arg399 and helix dipoles of the symmetrically arranged TM3 and TM10, rather than by precise molecular recognition.

\section{Data availability}

Cryo-EM density maps have been deposited in the Electron Microscopy Data Bank under the accession codes EMD-31757 (chloride-bound), 31758 (sulfate-bound) and 31759 (salicylate-bound). Atomic coordinates have been deposited in the Protein Data Bank under IDs 7V73 (chloride-bound), 7V74 (sulfate-bound) and 7V75 (salicylate-bound). The raw images have been deposited in the Electron Microscopy Public Image Archive, under accession code EMPIAR-xxxxx.

\section{Declarations}

\section{Acknowledgments:}

We thank the staff scientists at The University of Tokyo's cryo-EM facility, especially K. Kobayashi, T. Kusakizako, H. Yanagisawa, A. Tsutsumi, M. Kikkawa, and R. Danev.

\section{Funding:}

The MEXT Grant-in-Aid for Specially Promoted Research 16H06294 and JST CREST program 20344981 (to O.N.)

JSPS KAKENHI grant $17 \mathrm{H} 05000$ and $20 \mathrm{H} 03216$ (to T.N.)

AMED JP19am0101115 (support number 1111)

$\mathrm{NIH}$ grant DC017482 (to K.H.)

\section{Author contributions:}


H.F. prepared the cryo-EM samples. K.H. and S.T. performed the functional analyses. H.F. and T.N. collected and processed the cryo-EM data and built the structures. K.Y. assisted with data processing and structure refinement. T.S. and S.H. performed the MD simulation. H.F., T.N., K.H. and O.N. wrote the manuscript with input from the other authors. T.N., K.H. and O.N. supervised the research.

\section{Competing interests:}

O.N. is a cofounder and scientific advisor for Curreio. All other authors declare no competing interests.

\section{References}

1. WE Brownell, CR Bader, D Bertrand, Y. de R. Evoked mechanical responses of isolated cochlear outer hair cells. Science (80-.). 227, 194-196 (1985).

2. Zheng, J. et al. Prestin is the motor protein of cochlear outer hair cells. Nature 405, 149-155 (2000).

3. Liberman, M. C. et al. Prestin is required for electromotility of the outer hair cell and for the cochlear amplifier. Nature 419, 300-304 (2002).

4. Alper, S. L. \& Sharma, A. K. The SLC26 gene family of anion transporters and channels. Molecular Aspects of Medicine 34, 494-515 (2013).

5. Mistrík, P., Daudet, N., Morandell, K. \& Ashmore, J. F. Mammalian prestin is a weak Cl-/HCO3electrogenic antiporter. J. Physiol. 590, 5597-5610 (2012).

6. Franchini, L. F. \& Elgoyhen, A. B. Adaptive evolution in mammalian proteins involved in cochlear outer hair cell electromotility. Mol. Phylogenet. Evol. 41, 622-635 (2006).

7. Ashmore, J. F. Forward and reverse transduction in the mammalian cochlea. Neurosci. Res. Suppl. 12, S39-S50 (1990).

8. Santos-Sacchi, J. Reversible inhibition of voltage-dependent outer hair cell motility and capacitance. J. Neurosci. 11, 3096-3110 (1991).

9. Oliver, D. et al. Intracellular anions as the voltage sensor of prestin, the outer hair cell motor protein. Science 292, 2340-3 (2001).

10. Rybalchenko, V. \& Santos-Sacchi, J. Allosteric Modulation of the Outer Hair Cell Motor Protein Prestin by Chloride. in 116-126 (World Scientific Pub Co Pte Lt, 2003). doi:10.1142/9789812704931_0015

11. Rybalchenko, V. \& Santos-Sacchi, J. Anion control of voltage sensing by the motor protein prestin in outer hair cells. Biophys. J. 95, 4439-4447 (2008).

12. Cazals, Y. Auditory sensori-neural alterations induced by salicylate. Prog. Neurobiol. 62, 583-631 (2000).

13. Geertsma, E. R. et al. Structure of a prokaryotic fumarate transporter reveals the architecture of the SLC26 family. Nat. Struct. Mol. Biol. 22, 803-808 (2015).

14. Walter, J. D., Sawicka, M. \& Dutzler, R. Cryo-EM structures and functional characterization of murine Slc26a9 reveal mechanism of uncoupled chloride transport. Elife 8, (2019). 
15. Chi, X. et al. Structural insights into the gating mechanism of human SLC26A9 mediated by its Cterminal sequence. Cell Discov. 6, 55 (2020).

16. Canul-Tec, J. C. et al. Structure and allosteric inhibition of excitatory amino acid transporter 1. Nature 544, 446-451 (2017).

17. Oda, K. et al. Consensus mutagenesis approach improves the thermal stability of system xc transporter, XCT, and enables cryo-EM analyses. Protein Sci. 29, 2398 (2020).

18. Arakawa, T. et al. Crystal structure of the anion exchanger domain of human erythrocyte band 3 . Science (80-). 350, 680-684 (2015).

19. Lu, F. et al. Structure and mechanism of the uracil transporter UraA. Nat. 2011 4727342 472, 243246 (2011).

20. Chang, M.-H. et al. Slc26A9 - anion exchanger, channel and $\mathrm{Na}+$ transporter. J. Membr. Biol. 228, 125 (2009).

21. Dorwart, M. R., Shcheynikov, N., Wang, Y., Stippec, S. \& Muallem, S. SLC26A9 is a Cl - channel regulated by the WNK kinases. J. Physiol. 584, 333 (2007).

22. Rapp, C., Bai, X. \& Reithmeier, R. A. F. Molecular analysis of human solute carrier SLC26 anion transporter disease-causing mutations using 3-dimensional homology modeling. Biochim. Biophys. Acta - Biomembr. 1859, 2420-2434 (2017).

23. Gorbunov, D. et al. Molecular architecture and the structural basis for anion interaction in prestin and SLC26 transporters. Nat. Commun. 5, (2014).

24. Rybalchenko, V. \& Santos-Sacchi, J. Cl- flux through a non-selective, stretch-sensitive conductance influences the outer hair cell motor of the guinea-pig. Journal of Physiology 547, 873-891 (2003).

25. Tunstall, M. J., Gale, J. E. \& Ashmore, J. F. Action of salicylate on membrane capacitance of outer hair cells from the guinea-pig cochlea. J. Physiol. 485 (Pt 3), 739-52 (1995).

26. Kakehata, S. \& Santos-Sacchi, J. Effects of salicylate and lanthanides on outer hair cell motility and associated gating charge. J. Neurosci. 16, 4881-4889 (1996).

27. Shehata, W. E., Brownell, W. E. \& Dieler, R. Effects of Salicylate on Shape, Electromotility and Membrane Characteristics of Isolated Outer Hair Cells from Guinea Pig Cochlea. Acta Otolaryngol. 111, 707-718 (1991).

28. Homma, K. \& Dallos, P. Evidence that prestin has at least two voltage-dependent steps. J. Biol. Chem. 286, 2297-307 (2011).

29. Gorbunov, D., Hartmann, J., Renigunta, V. \& Oliver, D. A glutamate scan identifies an electrostatic switch for prestin activity. PS973 in 41st meeting of the Association for Research in Otolaryngology (San Diego, CA, Feb 10-14, 2018).

30. Oliver, D., Gorbunov, D., Hartmann, J., Lenz, D. \& Renigunta, V. An Electrostatic Switch for Gating the Electromechanical Activity of SLC26A5 (prestin). Biophys. J. 116, 169a (2019).

31. Drew, D. \& Boudker, O. Shared Molecular Mechanisms of Membrane Transporters. http://dx.doi.org/10.1146/annurev-biochem-060815-014520 85, 543-572 (2016). 
32. Homma, K., Duan, C., Zheng, J., Cheatham, M. A. \& Dallos, P. The V499G/Y501H mutation impairs fast motor kinetics of prestin and has significance for defining functional independence of individual prestin subunits. J. Biol. Chem. 288, 2452-2463 (2013).

33. Dallos, P. et al. Prestin-based outer hair cell motility is necessary for mammalian cochlear amplification. Neuron 58, 333-9 (2008).

34. Rajagopalan, L. et al. Tuning of the outer hair cell motor by membrane cholesterol. J. Biol. Chem. 282, 36659-70 (2007).

35. Fang, J., Izumi, C. \& Iwasa, K. H. Sensitivity of Prestin-Based Membrane Motor to Membrane Thickness. Biophys. J. 98, 2831-2838 (2010).

36. Izumi, C., Bird, J. E. \& Iwasa, K. H. Membrane Thickness Sensitivity of Prestin Orthologs: The Evolution of a Piezoelectric Protein. Biophys. J. 100, 2614-2622 (2011).

37. Chang, Y. N. et al. Structural basis for functional interactions in dimers of SLC26 transporters. Nat. Commun. 10, 1-10 (2019).

38. Carugo, O. Buried chloride stereochemistry in the Protein Data Bank. BMC Struct. Biol. 2014141 14, 1-7 (2014).

39. Dallos, P. \& Fakler, B. Prestin, a new type of motor protein. Nature Reviews Molecular Cell Biology 3 , 104-111 (2002).

40. Bai, J.-P. et al. Prestin's anion transport and voltage-sensing capabilities are independent. Biophys. J. 96, 3179-86 (2009).

41. Frolenkov, G. I., Atzori, M., Kalinec, F., Mammano, F. \& Kachar, B. The Membrane-based Mechanism of Cell Motility in Cochlear Outer Hair Cells. Mol. Biol. Cel/ 9, 1961-1968 (1998).

42. Kalinec, F., Holley, M. C., Iwasa, K. H., Lim, D. J. \& Kachar, B. A membrane-based force generation mechanism in auditory sensory cells. Proc. Natl. Acad. Sci. U. S. A. 89, 8671 (1992).

43. Kowarz, E., Löscher, D. \& Marschalek, R. Optimized Sleeping Beauty transposons rapidly generate stable transgenic cell lines. Biotechnol. J. 10, 647-653 (2015).

44. A. Kirchhofer, J. Helma, K. Schmidthals, C. Frauer, S. Cui, A. Karcher, M., Pellis, S. Muyldermans, C. S. Casas-Delucchi, M. C. Cardoso, H. Leonhardt, K. \& P. Hopfner, U. R. Modulation of protein properties in living cells using nanobodies. Nat. Struct. Mol. Biol. 17, 133-139 (2010).

45. Mastronarde, D. N. Automated electron microscope tomography using robust prediction of specimen movements. J. Struct. Biol. 152, 36-51 (2005).

46. Zivanov, J. et al. New tools for automated high-resolution cryo-EM structure determination in RELION3. Elife 7, (2018).

47. Rohou, A. \& Grigorieff, N. CTFFIND4: Fast and accurate defocus estimation from electron micrographs. J. Struct. Biol. 192, 216-221 (2015).

48. Zivanov, J., Nakane, T. \& Scheres, S. H. W. A Bayesian approach to beam-induced motion correction in cryo-EM single-particle analysis. IUCrJ 6, 5-17 (2019). 
49. Rosenthal, P. B. \& Henderson, R. Optimal determination of particle orientation, absolute hand, and contrast loss in single-particle electron cryomicroscopy. J. Mol. Biol. 333, 721-745 (2003).

50. Emsley, P., Lohkamp, B., Scott, W. G. \& Cowtan, K. Features and development of Coot. Acta Crystallogr. D. Biol. Crystallogr. 66, 486-501 (2010).

51. Bradley, P., Misura, K. M. S. \& Baker, D. Biochemistry: Toward high-resolution de novo structure prediction for small proteins. Science (80-). 309, 1868-1871 (2005).

52. Adams, P. D. et al. PHENIX: A comprehensive Python-based system for macromolecular structure solution. Acta Crystallogr. Sect. D Biol. Crystallogr. 66, 213-221 (2010).

53. Afonine, P. V. et al. Real-space refinement in PHENIX for cryo-EM and crystallography. Acta Crystallogr. Sect. D Struct. Biol. (2018). doi:10.1107/S2059798318006551

54. Yamashita, K., Palmer, C. M., Burnley, T. \& Murshudov, G. N. Cryo-EM single particle structure refinement and map calculation using Servalcat. bioRxiv 2021.05.04.442493 (2021). doi:10.1101/2021.05.04.442493

55. Murshudov, G. N. et al. REFMAC5 for the refinement of macromolecular crystal structures. Acta Crystallogr. Sect. D Biol. Crystallogr. 67, 355-367 (2011).

56. Kuwabara, M. F. et al. The extracellular loop of pendrin and prestin modulates their voltage-sensing property. J. Biol. Chem. 293, 9970-9980 (2018).

57. Wasano, K. et al. Systematic quantification of the anion transport function of pendrin (SLC26A4) and its disease-associated variants. Hum. Mutat. 41, 316-331 (2020).

58. Santos-Sacchi, J., Kakehata, S. \& Takahashi, S. Effects of membrane potential on the voltage dependence of motility-related charge in outer hair cells of the guinea-pig. J. Physiol. 510, 225-235 (1998).

59. Stuurman, N., Edelstein, A. D., Amodaj, N., Hoover, K. H. \& Vale, R. D. Computer Control of Microscopes using $\mu$ Manager. Curr. Protoc. Mol. Biol. CHAPTER, Unit14.20 (2010).

60. Schindelin, J. et al. Fiji - an Open Source platform for biological image analysis. Nat. Methods 9 , 676-682 (2012).

61. Šali, A. \& Blundell, T. L. Comparative Protein Modelling by Satisfaction of Spatial Restraints. J. Mol. Biol. 234, 779-815 (1993).

62. Davis, I. W. et al. MolProbity: all-atom contacts and structure validation for proteins and nucleic acids. Nucleic Acids Res. 35, W375-W383 (2007).

\section{Figures}


a

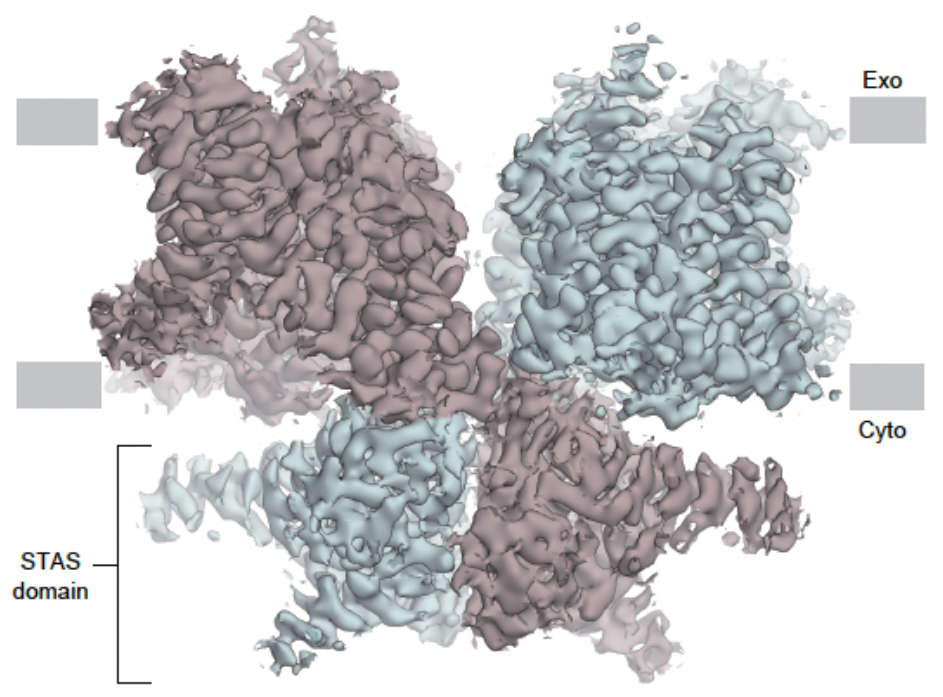

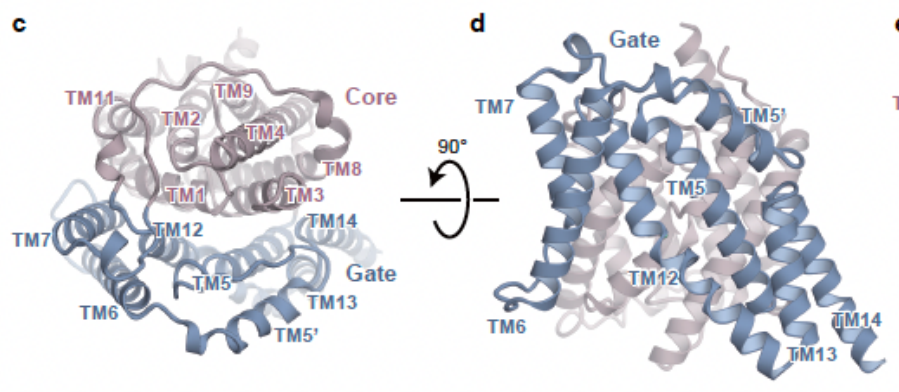
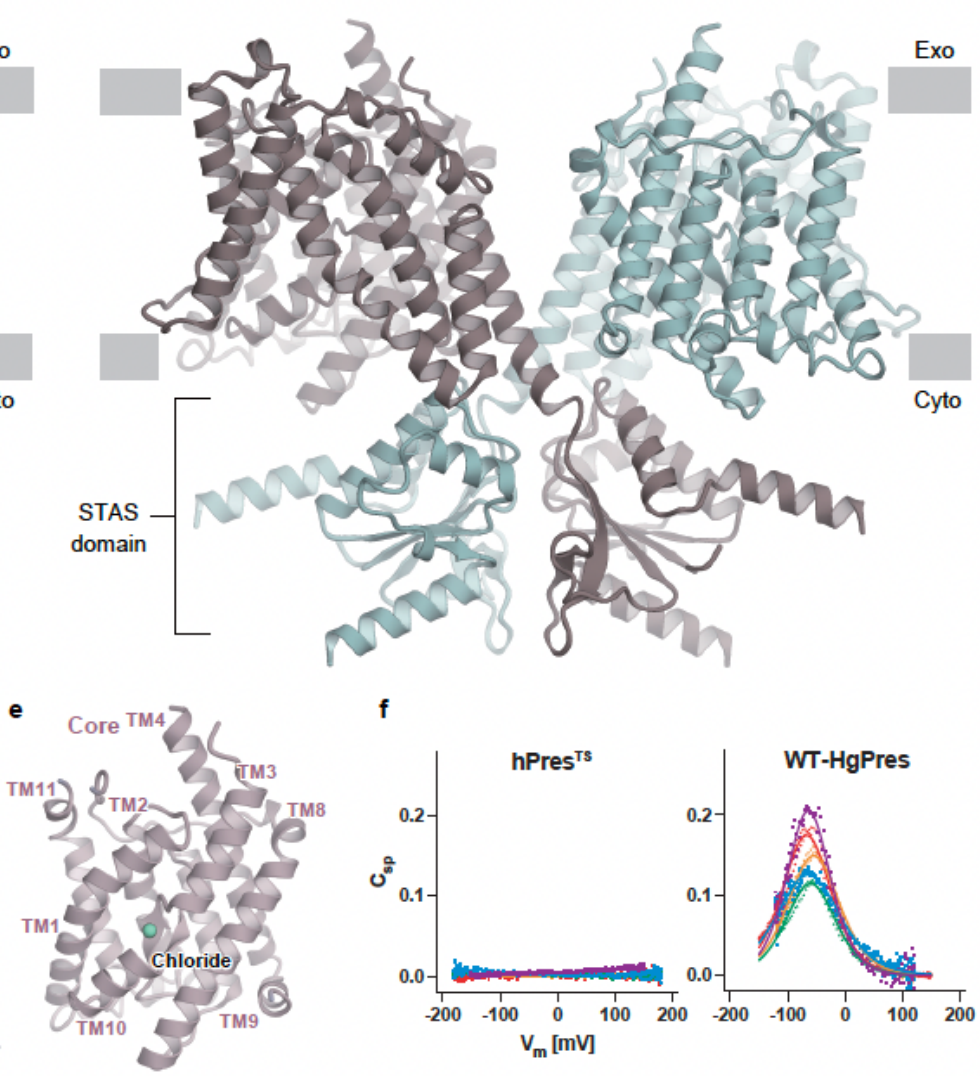

$f$

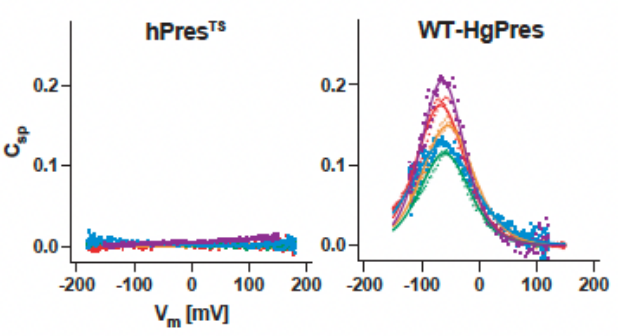

Figure 1

Cryo-EM structure of the hPresTS. (a) A Cryo-EM map of the hPresTS dimer. Each protomer is shown in a different color (green and dark brown). (b) Ribbon representation of the hPresTS dimer, with protomers shown in the same colors used in panel A. (c) Transmembrane domain of an hPresTS protomer, viewed from the extracellular side. The core (pink) and gate (blue) domains are shown as ribbon models. "TM" indicates transmembrane helix. See Supplementary Fig. 2 for the detailed TM numbering information. (d and e) Side views of the gate (d) and core (e) domains. The green sphere indicates the bound chloride ion. (f) Voltage-dependent cell membrane electric capacitance measurements in HEK293T cells expressing hPresTS (left panel) or wild-type naked mole rat prestin (HgPres, right panel). Five examples in different colors are shown for each protein. Solid lines (only for HgPres) indicate two-state Boltzmann fittings. The $a, V p k$, and Qmax values (mean \pm S.D.) were $0.032 \pm 0.01 \mathrm{mV}-1,-65 \pm 14 \mathrm{mV}$, and $12 \pm 5$ $\mathrm{fC} / \mathrm{pF}$, respectively $(n=22)$. NLC was undetectable in cells expressing hPresTS, although it targets the cell membrane (Supplementary Fig. 7). 
a

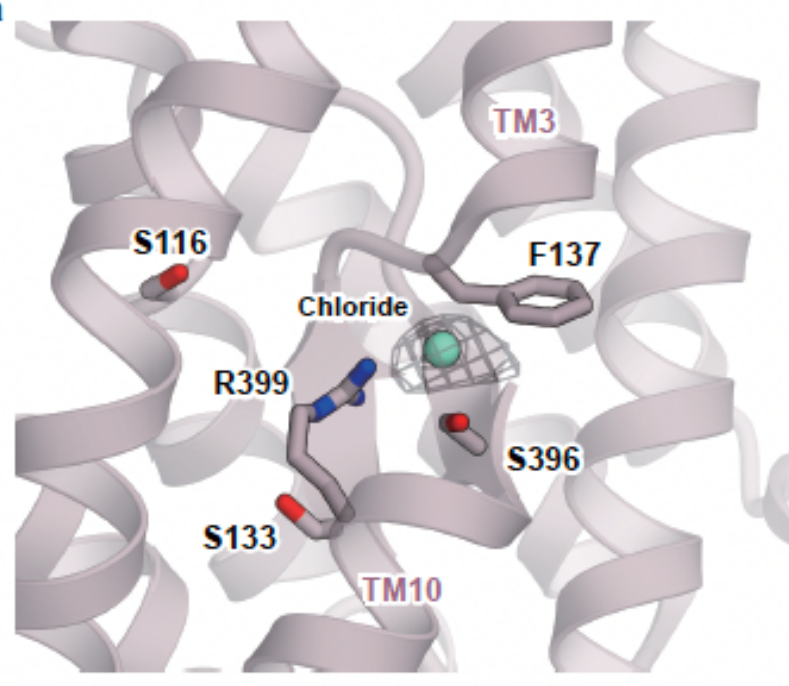

b

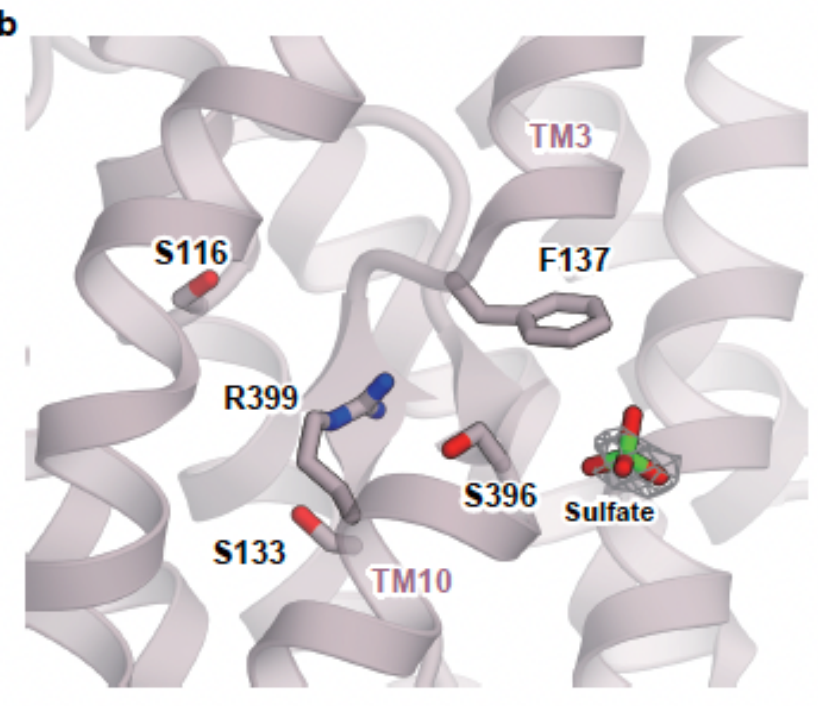

F137A
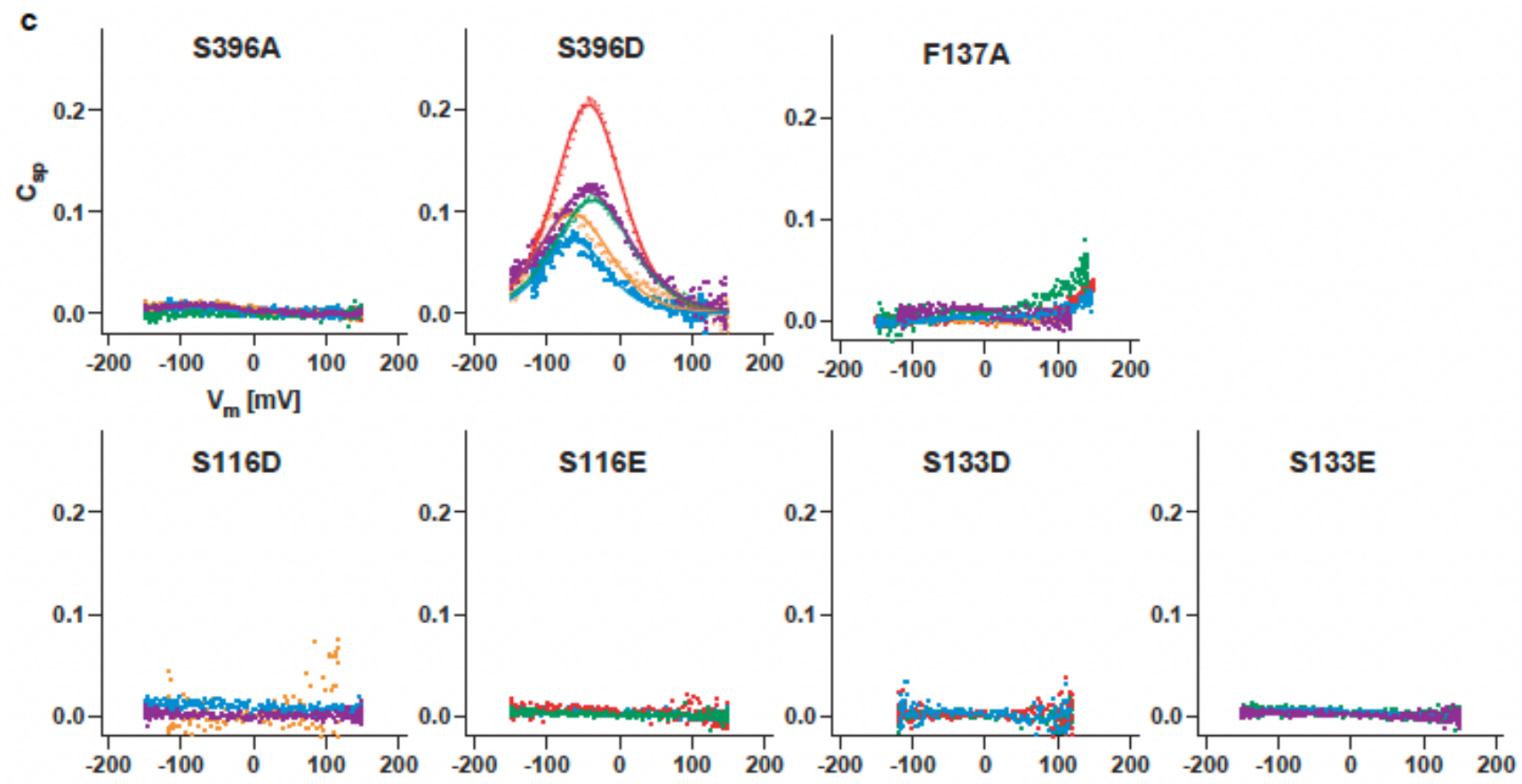

\section{Figure 2}

Anion binding sites of hPresTS. ( $a$ and $b$ ) Close-up views of the central anion binding pocket found in the chloride-bound (a) and sulfate-bound (b) hPresTS structures. Chloride and sulfate ions are indicated as a green sphere and a stick model, respectively. Residues that contribute to anion binding are also shown with stick models. The cryo-EM densities of the bound anions are indicated by gray meshes. (c) Cell membrane electric capacitance measurement in HEK293T cells expressing S396A-, S396D-, F137A-, S116D-, S116E-, S133D-, or S133E-HgPres. Three to five examples in different colors are shown for each panel. Solid lines (only for S396D-HgPres) indicate two-state Boltzmann fittings. The a, Vpk, and Qmax values (mean \pm S.D.) were $0.029 \pm 0.01 \mathrm{mV}-1,-51 \pm 11 \mathrm{mV}$, and $14 \pm 7 \mathrm{fC} / \mathrm{pF}$, respectively $(\mathrm{n}=7)$. These 
NLC parameters were statistically indistinguishable between WT vs. S396D (determined by one-way ANOVA followed by Dunnett's multi-comparison tests).
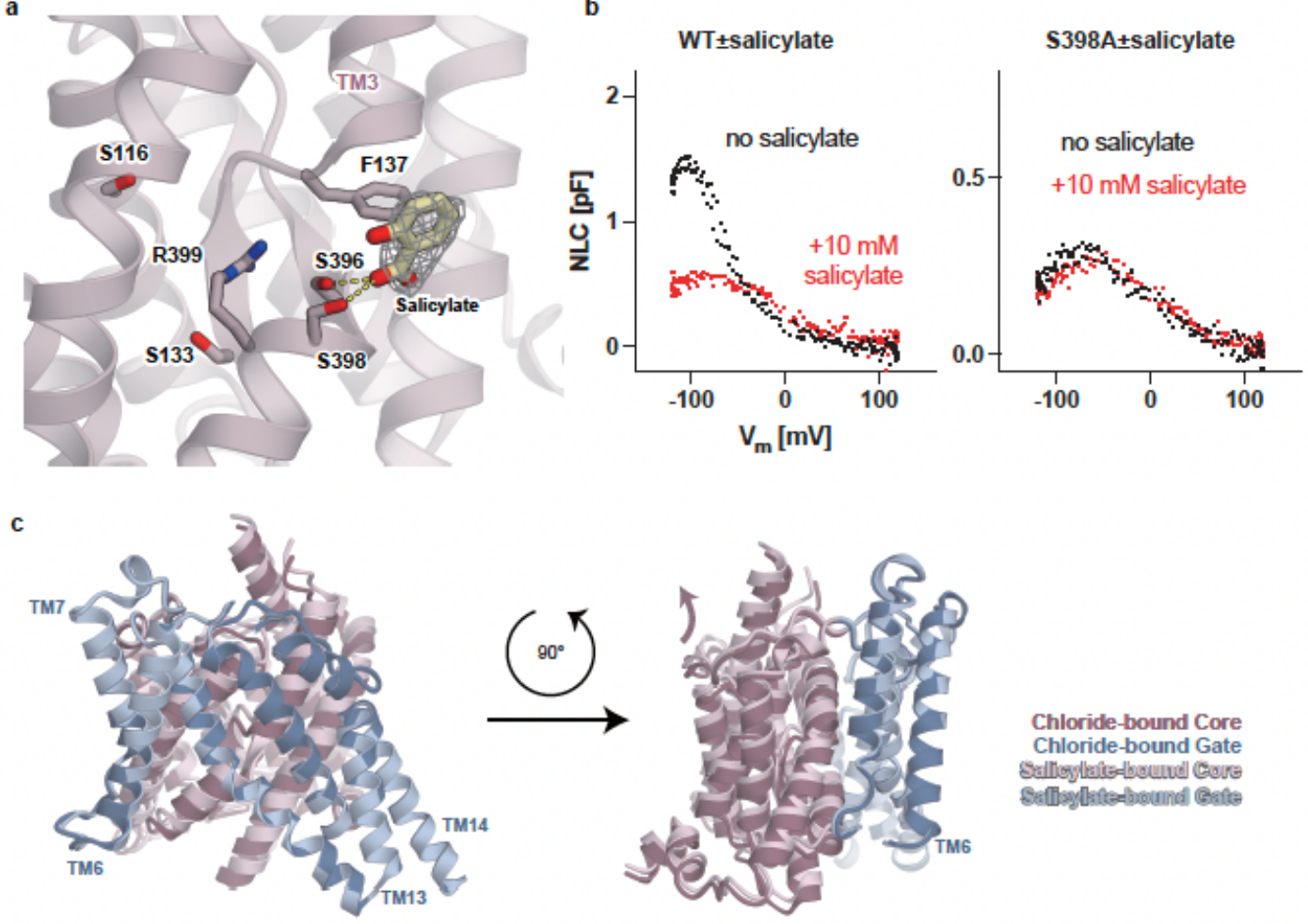

Chloride-bound Core

Chloride-bound Gate

Sallieylate-trowud Gove

Selliglatesound Gate

d
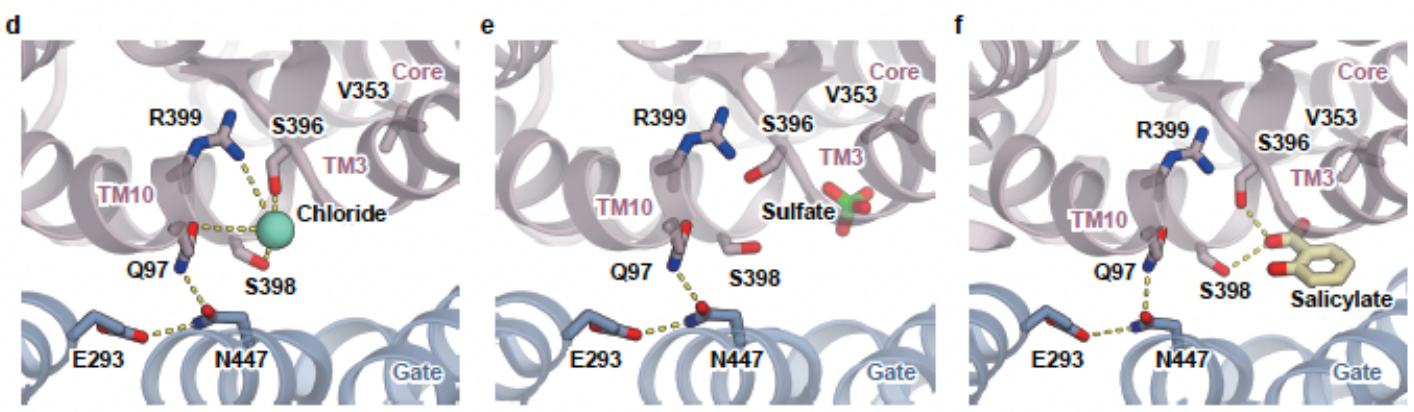

。
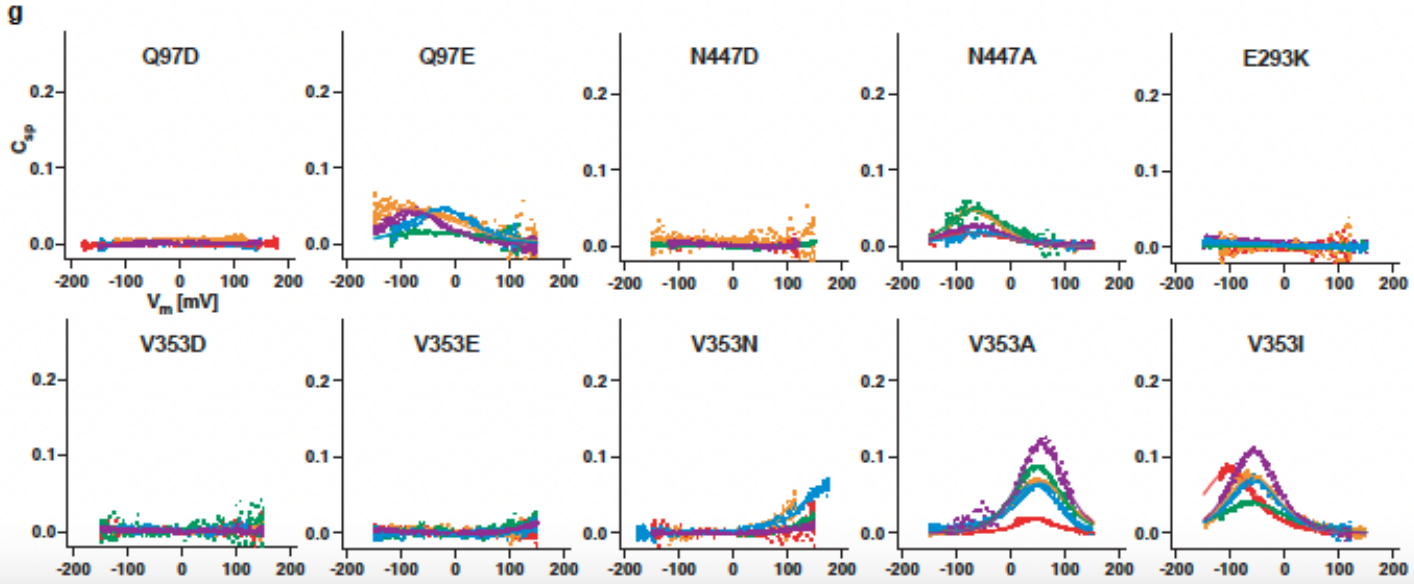

\section{Figure 3}

Salicylate binding and the interaction between the core and gate domains. (a) Close-up view of the central anion binding pocket of hPresTS with a bound salicylate. Salicylate and some important residues are shown in stick representations. The cryo-EM density of salicylate is shown as a gray mesh. Predicted 
short-distance interactions are indicated by yellow dotted lines. (b) The effect of salicylate on the NLC of WT (left) and S398A-HgPres (right). NLC was measured from the same cells before (black) and after (red) the application of $10 \mathrm{mM}$ salicylate in the bath solution. The total numbers of recordings were seven for both WT- and S398A-HgPres. (c) Rigid-body domain movement of the core domain with respect to the gate domain, as deduced from the chloride- vs. salicylate-bound hPresTS structures (shown in dark and light colors, respectively). See also Supplementary Movie 1. (d, e and f) The core-gate domain interface in the chloride-bound (d), sulfate-bound (e), and salicylate-bound (f) structures, viewed from the extracellular side. Putative hydrogen bonds are indicated by yellow dotted lines. (g) Cell membrane electric capacitance measurement in HEK293T cells expressing Q97D-, Q97E-, N447D-, N447A-, E293K-, V353D-, V353E, V353N-, V353A-, and V353I-HgPres. Four to five examples in different colors are shown for each panel. Solid lines (for Q97E-, N447A-, V353A-, and V353I-HgPres) indicate two-state Boltzmann fittings. The $a$, Vpk, and Qmax values (mean \pm S.D.) were as follows: [0.021 $\pm 0.01 \mathrm{mV}-1,-59 \pm 30 \mathrm{mV}, 7.5 \pm 3.3$ $\mathrm{fC} / \mathrm{pF}]$ for Q97E $(\mathrm{n}=4)$; $[0.027 \pm 0.001 \mathrm{mV}-1,-65 \pm 8 \mathrm{mV}, 4.4 \pm 1.7 \mathrm{fC} / \mathrm{pF}]$ for N447A $(\mathrm{n}=5) ;[0.036 \pm 0.01$ $\mathrm{mV}-1,51 \pm 8 \mathrm{mV}, 8.4 \pm 3.7 \mathrm{fC} / \mathrm{pF}]$ for V353A $(\mathrm{n}=6)$; and [0.031 $\pm 0.01 \mathrm{mV}-1,-63 \pm 20 \mathrm{mV}, 8.2 \pm 4.0 \mathrm{fC} / \mathrm{pF}]$ for V353I $(n=6)$.
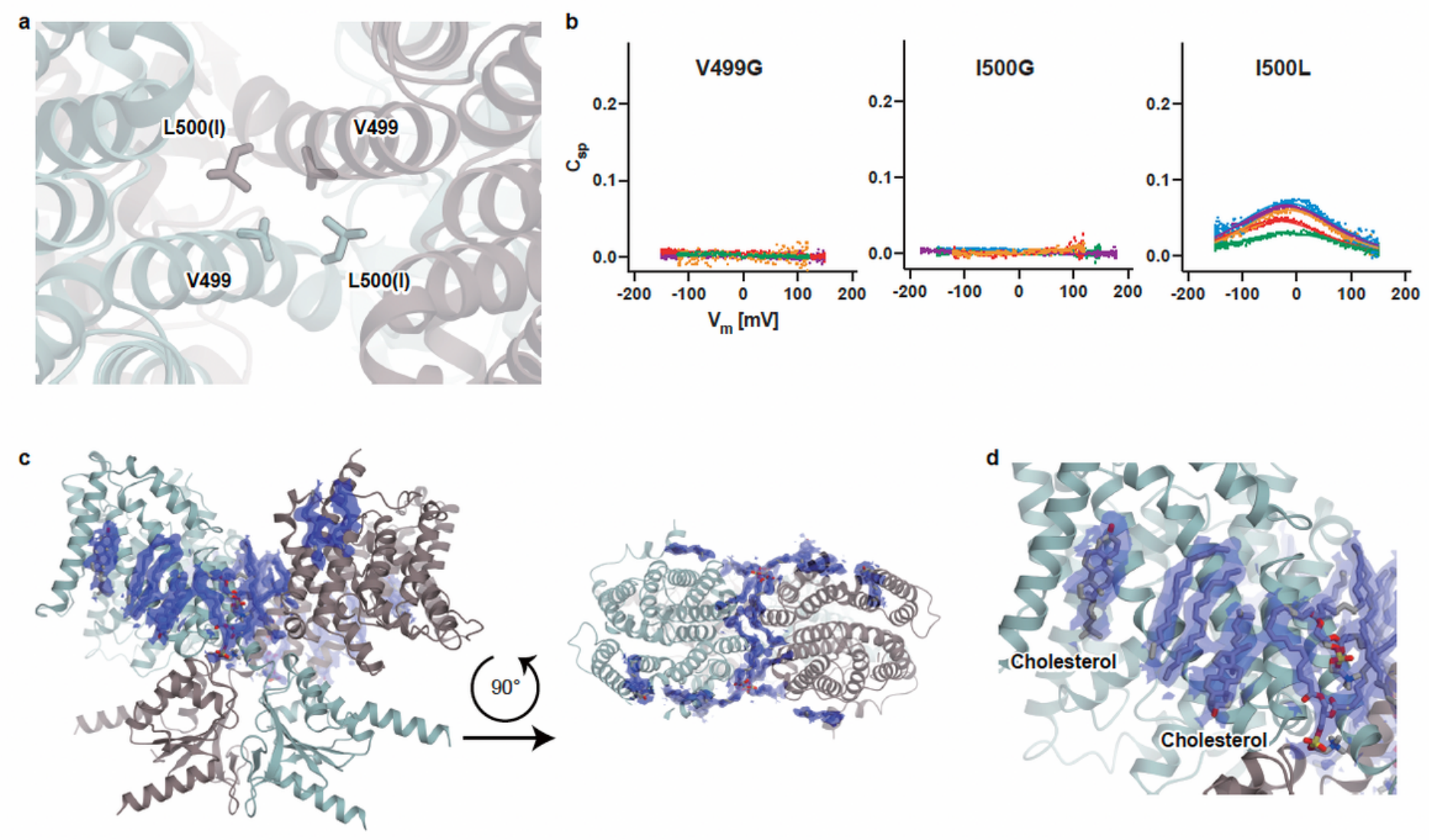

\section{Figure 4}

Dimerization of hPresTS. (a) Hydrophobic interactions are mediated by Val499 and Leu500 (lle500 in wild-type) at the C-termini of the TM14 helices. (b) Cell membrane electric capacitance measurements in HEK293T cells expressing V499G-, I500G-, and I500L-HgPres. Five examples in different colors are shown for each panel. Solid lines (only for $1500 \mathrm{~L}-\mathrm{Hg}$ Prestin) indicate two-state Boltzmann fittings. The a, Vpk, 
and Qmax values of I500L-HgPres (mean \pm S.D.) were $0.017 \pm 0.01 \mathrm{mV}-1,-22 \pm 8 \mathrm{mV}$, and $13 \pm 4 \mathrm{fC} / \mathrm{pF}$, respectively $(n=5)$. (c) Lipids found on the hPresTS structure. Phospholipids and cholesterols are shown as gray stick models with cryo-EM densities (blue). (d) Close-up view of lipids found at the dimerization interface. Phospholipids and cholesterols are shown as stick models with cryo-EM densities (blue).

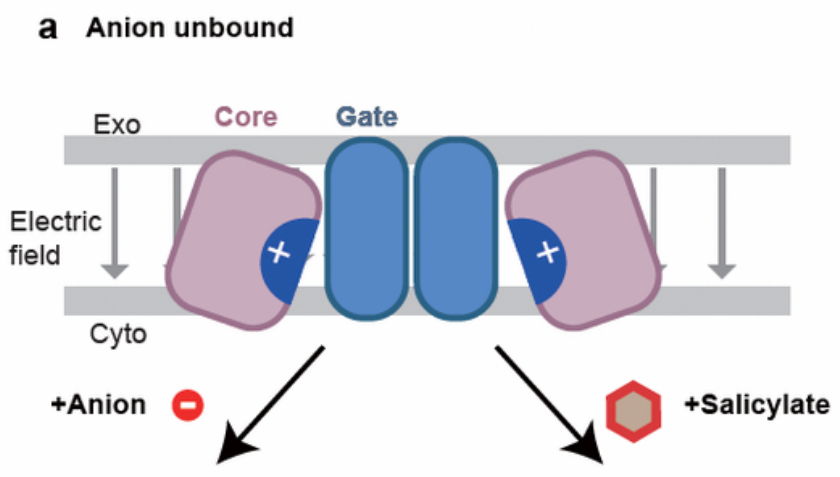

b Anion bound

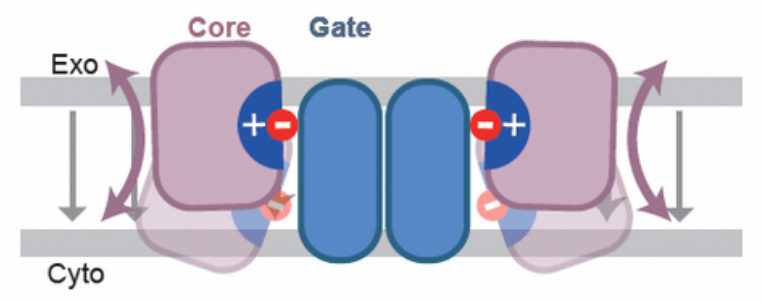

C Salicylate bound

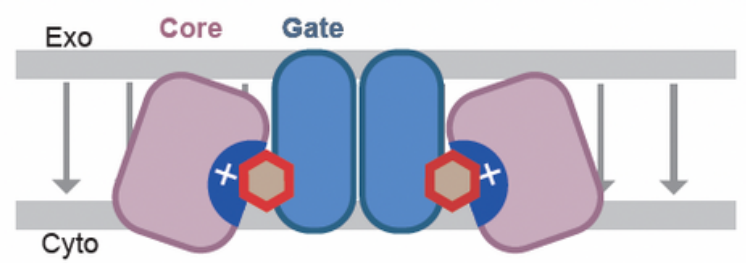

\section{Figure 5}

Hypothetical elevator-like movement of the core domains facilitated or inhibited by anion binding. The highly positively charged central pocket (indicated by " + ") is forcibly exposed toward the intracellular solvent in the absence of an anion (a). Anion binding allows the core domain to adopt the occluded state (b). Salicylate binds to the positively charged central pocket, but sterically inhibits the transition from the inward-open to the occluded state (c). Despite the apparent charge neutralization of the pocket, there is a complex charge bias in the micro-environment of the anion site, due to the helix dipoles of TM3 and TM10. This allows the voltage-dependent conformational change of the core domain, which generates the charge transfer (NLC).

\section{Supplementary Files}

This is a list of supplementary files associated with this preprint. Click to download.

- Supplementarynat.pdf

- ValidationReports.pdf

- ReportingsummaryPrestin2.pdf

- Movie1.mov 
- Movie2.mov

Page 23/23 